\title{
Complexity, evaluation and the effectiveness of community-based interventions to reduce health inequalities
}

Anna Matheson, Kevin Dew and Jackie Cumming

\section{Introduction}

...if the evidence was overwhelming - not just as to the importance of socioeconomic factors for health, which is overwhelming, but as to the effectiveness of specific and feasible interventions - then the opposing interests labout intervention approaches] could probably be overcome. ${ }^{1}$

Reducing health inequalities has been part of the New Zealand government's agenda since the early 1990s. ${ }^{2}$ Analysis of health inequalities has emphasised geographic area as it has been shown, through various measures of socio-economic position, that there is an enduring relationship between geography and health outcomes. However, the nature of this relationship is much debated. ${ }^{3,4}$ As a consequence, the 'community' - most often defined by geographic area - has become popular as a site for interventions to reduce health inequalities. Unfortunately, and as Evans notes above, the evidence of the effectiveness of these interventions is not overwhelming. ${ }^{5,6}$ Each of a handful of systematic reviews that attempts to synthesise the evidence cites difficulties in the practicalities of collecting, collating and comparing the evidence. $^{7-10}$ As a result it has been argued that systematic reviews of the effectiveness of health inequalities interventions, using conventional protocols of the hierarchies of evidence, are inadequate for locating and then comparing, studies and their findings. ${ }^{10,11}$

That many of the interventions discussed in the literature are regarded as having inadequate evaluations of their effectiveness is a central issue that informs this evidence base. Findings of evaluations are often limited both because of the difficulties in attributing particular health outcomes to a particular intervention, as well as the fact that many evaluations are inadequately resourced..$^{10,12-14}$ This paper argues that there are inherent difficulties in being able to predict the end result of a course of action that aims to affect long-term outcomes such as health inequalities, and that having a better understanding of the processes of complexity should enable insight into how social processes may influence such an outcome. The research described

\section{Abstract}

Reducing health inequalities has been part of the New Zealand government's agenda since the early 1990s. As a result, interventions have been implemented nationally with the explicit goal of reducing health inequalities. This paper describes findings from a comparative case study of two community-based interventions - carried out in different New Zealand communities. Complexity theory was used as an analytic tool to examine the case data, and provided a systematic way in which to explore 'local' issues by taking a 'whole system' perspective. The findings showed that two important influences on the successful implementation of the interventions were the existence and capacity of local organisations and their relationships with government agencies. The analysis provided a dynamic picture of shared influences on the interventions in different communities and in doing so offered insight into intervention effectiveness. It is argued in this article that, for examining intervention effectiveness, it is essential to have a theoretical understanding of the behaviour of the complex system in which they are implemented. This theoretical understanding has implications for the appropriate design of interventions to reduce health inequalities, and in turn should lead to more meaningful ways to evaluate them.

Key words: community interventions, health inequalities, complexity theory, case studies, evaluation.

Health Promotion Journal of Australia 2009; 20:221-6

\section{So What}

Tangible evidence of the effectiveness of interventions to reduce health inequalities is hard to come by. Many argue that better theoretical and methodological tools are needed to generate and understand the evidence. Complexity theory provides tools for a systematic analysis of the processes of social change that are central to the practice of health promotion and for understanding the social mechanisms that sustain health inequalities. 
here explores the implementation of two community-based interventions - carried out in different communities - through the perspective of complexity theory. The findings suggest that this theoretical approach has the potential to inform how community interventions can be more effective as well as suggesting more appropriate ways to evaluate them.

\section{Methods}

The study design was a comparative case study of two community-based interventions that aimed, among other things, to reduce health inequalities. ${ }^{15}$ The interventions were the Housing, Insulation and Health Study ${ }^{16}$ and the Intersectoral Community Action for Health project. ${ }^{17}$ The interventions were carried out in 2000 and 2005, with data collection occurring between 2004 and 2005. The data were collected for each intervention sequentially and a snowballing technique was used - within each case - to identify relevant data sources. The sources were primarily documentation relating to the interventions and in-depth interviews with key informants from varied organisations. The participant organisations included government agencies and local organisations within six New Zealand communities - Otara, Nuhaka, Mahia, Kapiti, Poriua and Christchurch (see Figure 1) - across which the two projects were conducted.

How a case is framed is central to a research approach informed by complexity. ${ }^{18-20}$ Often the boundaries to a case are self-evident - for example, viewing a person as representing a case of a disease - however, the framing of a case becomes less immediately apparent when the case begins to incorporate

\section{Figure 1: Map of New Zealand with geographic locations of} community interventions.

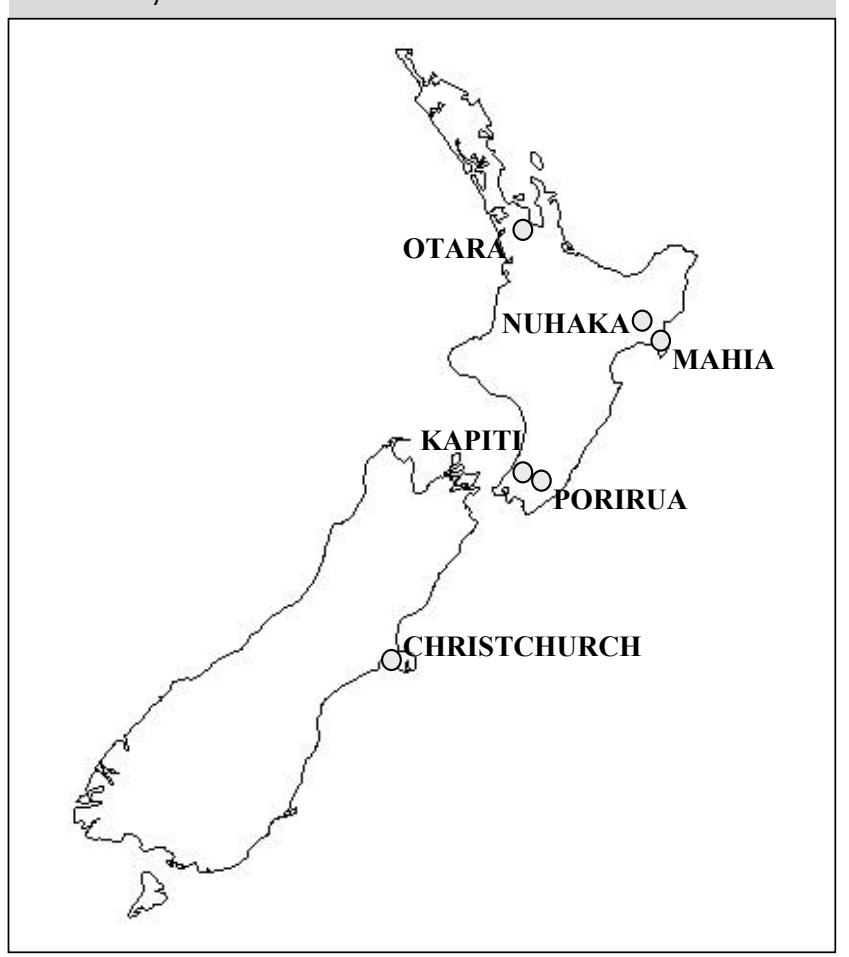

numbers of diverse individuals, organisations and social groupings. Both cases of an intervention selected for this research were New Zealand community-based interventions that had an aim to reduce health inequalities; had a focus on local action; utilised similar intervention approaches in different geographic locations, and involved a number of organisations in their conception and implementation.

For the purposes of comparison, the six geographic areas in which the projects were conducted have been rated on one scale of socio-economic inequality - the New Zealand Deprivation Index (NZDep06). ${ }^{21}$ Two of the areas, Christchurch and Kapiti, are rated as having relatively low deprivation and better health outcomes in relation to the other four. Otara, Porirua, Nuhaka and Mahia have relatively high deprivation on the NZDep06 scale and therefore were indicated as suffering worse health outcomes (see Table 1). The data were analysed using theory elaboration ${ }^{22}$ whereby cases are illuminated through applying prior theoretical understandings - in this instance complexity theory.

\section{Complexity theory}

In the past decade complexity theory has become increasingly utilised within the health and social sciences. Indeed some have described these recent developments as the 'complexity turn', and argue that complexity theory might offer a new framing for empirical studies into diversity, social change and inequality. ${ }^{23-26}$ It is suggested that as societies become more populated and more interconnected, there is an even greater need to understand the processes of complexity in order to gain insight into the causes of the emergent social patterns - such as health inequalities. ${ }^{27-29}$ The ability to describe and depict these social patterns has become ever more sophisticated in many developed countries over the past 25 years. ${ }^{1}$ With this knowledge has come an increased understanding of the extent to which the activities of our social systems are interrelated. Many argue, however, that the development of theoretical and methodological tools to discern the nature of these interrelations is still limited.

Table 1: New Zealand Deprivation Index Score of the six communities in which case studies were undertaken.

\begin{tabular}{lcc}
\hline Case & Area & NZDep06 Score \\
\hline HIHS & Otara & 10 \\
HIHS & Nuhaka & 9 \\
HIHS & Mahia & 10 \\
ICAH & Porirua & 10 \\
ICAH & Kapiti & 5 \\
HIHS & Christchurch & 5 \\
\hline Notes: & \\
a) Indicative scores given that study communities did not map to exactly to NZDep areas \\
(1 = least deprived 10 = most deprived). \\
Source: White P, Gunston J, Salmond C, Atkinson J, Crampton P, 2008. Atlas of \\
Socioeconomic Deprivation in New Zealand NZDep2006. Wellington: Ministry of Health.
\end{tabular}


At the core of complexity theory is the notion that individual properties differ from collective properties. This resonates strongly with the influential epidemiological principle put forth by Geoffrey Rose that "causes of individual cases are not the same as the causes of overall incidence". ${ }^{1}$ While concurring with this principle, complexity theory adds further depth to this understanding. The theory holds that this difference results from the interactions that occur between and among parts of a collective, between and among parts and collectives, and between and among collectives. The theoretical picture this creates is one of overlapping systems that have some coherence but that are also linked to, and part of, other systems that are continually adapting to each other. This focus on the interrelationships of a complex system as being central to causal processes, gives reason to think that complexity theory may hold some useful ways to think about complex social processes.

There is a growing literature that argues the need to consider social interventions as occurring within a complex system. ${ }^{30-32}$ A central tenet of the theory is that long-term futures will always be uncertain. This view highlights the importance of having knowledge about the nature of the outcomes or goals being addressed. For example, there is a difference between a population-level outcome such as inequalities in health outcomes and more specific short-term intervention goals - they are, however, related. Indeed, some have suggested that complexity theory's perspective on outcomes has important implications for how interventions are evaluated. Sheill and colleagues for example, argue that settings such as schools, hospitals and primary care are themselves complex systems. ${ }^{30}$ Therefore evaluations of interventions in those settings should consider them as sets of dynamic relationships. ${ }^{33}$

\section{Case Study 1: Housing, Insulation and Health Study}

The first case was a community-based randomised, controlled trial of the health impacts of retrofitting insulation into houses that previously had no insulation. The Housing, Insulation and Health Study (HIHS) was carried out in seven communities around New Zealand, with researchers working in partnership with local organisations in each area. From those organisations, four - in Otara, Nuhaka, Mahia and Christchurch - were selected to participate in the research presented in this paper. The local organisations comprised two non-governmental community health organisations, a local Māori (the indigenous people of Aotearoa/New Zealand), health provider and a government public health unit.

The intention of the HIHS was to prove, through application of a rigorous research design, that insulating people's homes improved aspects of their health, and that targeting a more vulnerable population had the potential to reduce health inequalities. The HIHS was successful in terms of a number of its intentions, including: achieving high response rates for people participating, and remaining, in the study for each area; numbers of houses being insulated; carrying out advocacy for more resources to insulate homes in New Zealand; applying a robust research design to understand the data; and publishing the results in high-profile scientific journals. ${ }^{16,34}$

\section{Case Study 2: Intersectoral community action for health projects}

The second case was the Intersectoral Community Action for Health projects (ICAH) that resulted in the establishment of ICAH projects in four New Zealand communities - the Far North, South Auckland, Kapiti and Porirua. The overall aims of the ICAH initiative were set by a central government agency to encompass similar characteristics of four preexisting community projects in these areas. These aims were to: improve health outcomes in the community, particularly for Māori, Pacific peoples, and other population groups with poorer outcomes; establish intersectoral relationships for community action; capture and incorporate local knowledge; and use the knowledge gained to inform the work of the regional health provider.

The ICAH projects in Porirua and Kapiti were selected for this research. In these locations, local organisations charged with co-ordinating community health activities were established as a key part of the intervention. Key informants from 11 organisations involved in the ICAH intervention - including national, regional, local government, community health and health service provider organisations - participated in this research. An earlier evaluation of the ICAH projects found successes in the organisation of community activities but the evaluation timeframe was too short to show long-term impacts such as improving the relative health status of the communities. ${ }^{17}$

\section{Findings: government and community distance}

The policy environment influenced implementation of the interventions in two ways. The first of these involved the policy areas that allowed the interventions to become realities in the first instance. The centre-left Labour coalition government elected in 1999 introduced a policy focus on long-term outcomes, such as reducing inequalities. They also introduced relational and local methods of intervention such as community/government partnerships, whole of government approaches, intersectoral action and the devolution of decision-making to local areas within the health system. Both of the study interventions were able to take place because of this wider environment that made acceptable their goals, outcomes and methods. The second influence, discussed here, arose from policy initiatives that led to both the creation, 
and the strengthening, of local organisations that had a mandate to participate in the interventions.

The selected case interventions were different in many ways but, for both, the involvement of local organisations was critical to their implementation. The HIHS was research initiated, and utilised community organisations to carry out the research in their area. The ICAH was government agency initiated and utilised both existing and newly created community organisations to carry out the intervention aims. The goals and subsequent trajectories of each intervention were therefore different, however, the research found many similarities in the manner in which the wider context impacted the implementation of the interventions. A recurring theme throughout the case studies was that of 'distance'. This distance is sometimes related to geographic distance but in most instances related to social and cultural distance. Those key informants that reported their communities as distant from the centre were also those that had high deprivation according to the NZDep06 score.

For the HIHS intervention the very existence, within the participating communities, of local organisations with a mandate and capacity to participate was essential to those communities being involved in the study. They were also key to the successful completion of the HIHS through expediting the recruitment and retention of research participants. A significant impediment to the capacity of the local organisations was found to be the competitive, uncoordinated and short-term processes related to their funding sources. These processes diverted time and energy away from the intent of these organisations. The case findings showed that the historical government policy directions and initiatives, such as past funding arrangements, also influenced current capacity.

Those communities that were geographically or socially, and culturally, distant, reported that this distance impacted on their relationships with government agencies. This put them at a disadvantage in many formal and informal processes through there being greater distrust, limited knowledge of government processes, and having few influential relationships. For the local organisation in Otara - a community that can also be characterised as culturally diverse - this situation was compounded by intense competition with other local organisations for both community voice and resources.

The ICAH case findings resonated with those of the HIHS. Findings highlighted that local ICAH organisations' ability to progress their goals was impacted by the degree of responsiveness from government agencies to information that came from the ICAH activities. Well-supported communityneeds reports were produced through a collaborative process. At the outset of the ICAH initiatives these reports, which contained diverse forms of evidence, had considerable community involvement and commitment, and ownership from the government agencies. As time progressed the government agencies' processes to deal with information coming from the $\mathrm{ICAH}$ projects, such as those needs reports, changed. It was apparent that, within the two main central agencies, minimal mechanisms existed for monitoring, and acting upon, the information that was generated, despite dedicated community liaison positions having been created.

This uncoordinated and unresponsive formal engagement between the ICAH interventions and the government agencies impacted differently on the two communities. The importance of being distant from the centre was captured again in this case study. Kapiti was considered both relatively culturally homogenous as well as 'close' to the centre. The impacts of this could be seen through the experiences of the Kapiti ICAH organisation wherein they already had existing credibility in providing a voice for the community, significant relationships and knowledge of government processes, and ways of operating that were similar to the government agencies funding them. In Porirua, which is culturally diverse as well as distant, the ICAH organisation experienced intense competition for resources and community voice, they had less knowledge and experience of government processes, and their interactions with government agencies were frequently portrayed through the findings as being characterised by friction and conflict.

In contrast to Kapiti, the Porirua ICAH was much less confident in its credibility as a community voice, and indeed recognised the many competing perspectives operating within Porirua. The findings also showed that this was reflected in the less clear, and less certain, aims of the Porirua ICAH. Key informants from Kapiti expressed confidence about their ability to influence the government agencies, and expressed a high degree of trust in those agencies. On the other hand, Porirua informants expressed a resignation around their inability to influence, and a high degree of distrust of government agencies. It is also illustrative to highlight that Porirua contained striking differences in socioeconomic position within its local government boundaries. It was expressed throughout the interviews that this situation constrained the voice of this already disadvantaged area to be acted upon within local government. This disadvantage to Porirua seems significant, given other limits - related to the interactions with government and the internal dynamics of the community - to opportunities for individuals and groups that were highlighted through examining the progress of the $\mathrm{ICAH}$ interventions. The Porirua situation and the limits to opportunities for actionable voice were similar to those for the relatively deprived and culturally diverse Otara (as reported in the first HIHS case). 
The comparative analysis of both cases captured some shared dynamic influences. This wider context of relationships described above, which differed by community, was a critical feature in maintaining the intervention towards its goals. It was found that two important influences on the implementation of both interventions were the existence and capacity of local organisations, and their relationships with government agencies. Local organisations with the capacity and mandate to act, as well as their ability to connect with central organisations, were found to be crucial. The responsiveness of government agencies as well as the co-ordination of policy processes, such as contracting, monitoring and evaluation, impacted on the capacity of local organisations. For the more deprived communities the role of local organisations was even more important for taking up opportunities and for being a conduit to access knowledge and resources.

\section{Discussion: complexity theory, intervention and evaluation}

...examining the process of how community-based interventions improve health may be just as critical if not more important than evaluating the outcomes of community interventions. ${ }^{5}$

From a complexity perspective, the reason that the above quote may ring true is because of the rule of uncertainty. While long-term futures will always be uncertain, the best that can be done is to understand the processes that may impact these futures. And it is here, in understanding social processes, that complexity theory offers insights. The theory prioritises relationships in causal processes through its understandings of system trajectories, emergence, self-organisation, adaptation, feedback and sensitivity to initial conditions. ${ }^{35-37}$ And it is through these concepts that a useful way to explore 'local' issues by taking a 'whole system' perspective can be found.

The findings resonate with insights from many recent reviews and evaluations of community-based interventions. There seems to be consensus for greater recognition of: the importance of local context; the influence of different social levels; the role of relationships; and the nature of processes of change..$^{6,12-14,38}$ Complexity theory, as yet, has an unrealised potential for integrating these concerns. Taking a complexity approach to theorising interventions highlights elements of the wider system that may impact have an impact on interventions - including the role of government agencies themselves and the way that evaluation processes feed into their other policy processes.

Shiell and colleagues ${ }^{30}$ describe the difference between complex interventions and the complex system in which interventions are implemented. Settings such as schools, hospitals and primary care, as well as communities, are themselves set within a wider complex system that influences their activities. It is argued in this paper that it is essential to have a theoretical understanding of the behaviour of the "complex system in which interventions are implemented." This theoretical understanding has implications for the appropriate design of interventions to reduce health inequalities, and in turn should lead to more meaningful ways to evaluate them.

\section{Acknowledgements}

The authors would like to thank the research participants for the time and energy they gave to the research. They would also like to acknowledge Ilse Seilis and Mat Walton for valuable comments on drafts of the paper as well as financial support from the Health Research Council of New Zealand, by means of a PhD scholarship through He Kainga Oranga/Housing and Health Research Programme, University of Otago, Wellington.

\section{References}

1. Evans R. Interpreting and Addressing Inequalities in Health: from Black to Acheson to Blair...? London (UK): Office of Health Economics; 2002.

2. Matheson A, Dew K. Health, politics and justice. In: Dew K, Matheson A, editors. Understanding Health Inequalities in Aotearoa New Zealand. Dunedin (NZ): University of Otago Press; 2008.

3. Macintyre S, Ellaway A, Cummins S. Place effects on health: how can we conceptualise, operationalise and measure them? Soc Sci Med. 2002;55:12539.

4. Pearce J, Tisch C, Barnett R. Have geographical inequalities in cause-specific mortality in New Zealand increased during the period 1980-2001? N Z Med J. $2008 ; 121$

5. Atienza A, King A. Community-based health intervention trials: an overview of methodological issues. Epidemiol Rev. 2002;24(1):72-9.

6. Sorensen G, Emmons K, Hunt MK, Johnston D. Implications of the results of community intervention trials. Annu Rev Public Health. 1998;19:379-416.

7. Oglivie D, Hamilton V, Egan M, Petticrew M. Systematic reviews of health effects of social interventions: 1. Finding the evidence: how far should you go? J Epidemiol Community Health. 2005;59:804-8.

8. Petticrew M, Whitehead M, Macintyre S, Graham H, Egan M. Evidence for public health policy on inequalities: 1 : The reality according to policymakers. J Epidemiol Community Health. 2004;58:811-6.

9. Oglivie D, Egan M, Hamilton V, Petticrew M. Systematic reviews of health effects of social interventions: 2. Best available evidence: how low should you go? J Epidemiol Community Health. 2005;59:886-92.

10. Asthana S, Halliday J. Developing an evidence base for policies and interventions to address health inequalities: the analysis of "public health regimes". Milbank Q. 2006;84(3):577-603.

11. Pawson R, Greenhalgh T, Harvey G, Walshe K. Realist review - a new method of systematic review designed for complex policy interventions. J Health Serv Res Policy. 2005;10 Suppl 1:55-6.

12. Judge K, Bauld L. Learning from policy failure? Health Action Zones in England. Eur J Public Health. 2006;16(4):341-4.

13. Bauld L, Judge K, Barnes M, Benzeval M, MacKenzie M, Sullivan H. Promoting social change: the experience of the Health Action Zones in England. J Soc Policy. 2005;34(3):427-45.

14. O'Dwyer L, Baum F, Kavanagh A, Macdougal C. Do area-based interventions to reduce health inequalities work? A systematic review of evidence. Critical Public Health. 2007; 17(4):317-35.

15. Matheson A. Treating Communities for Health Inequalities: Complexity Matters. Wellington (NZ): University of Otago; 2008.

16. Howden-Chapman P, Matheson A, Crane J, Viggers H, Cunningham M, Blakely T, et al. Effect of insulating existing houses on health inequality: cluster randomised study in the community. BMJ. 2007;334:460.

17. Ministry of Health. Intersectoral Community Action for Health (ICAH) Evaluation: An Overview. Wellington (NZ): Ministry of Health; 2008.

18. Midgley G. Systemic Intervention: Philosophy, Methodology, and Practice - Contemporary Systems Thinking. London (UK): Kluwer Academic / Plenum Publishers; 2000 
19. Byrne D. Complexity, configurations and cases. Theory, Culture and Society. 2005;22(5):95-111.

20. Ragin C, Becker H. What is a Case? Exploring the Foundations of Social Inquiry. New York (NY): Cambridge University Press; 1992.

21. White P, Gunston J, Salmond C, Atkinson J, Crampton P. Atlas of Socioeconomic Deprivation in New Zealand NZDep2006. Wellington (NZ): Ministry of Health; 2008.

22. Vaughan D. Theory elaboration: the heuristics of case analysis. In: Ragin C, Becker A, editors. What is a Case? Exploring the Foundations of Social Inquiry. New York (NY): Cambridge University Press; 1992.

23. Walby S. Complexity theory, globalisation and diversity. British Sociological Conference; 2003 April; York,UK: University of York.

24. Gatrell A. Complexity theory and geographies of health: a critical assessment. Soc Sci Med. 2005;60:2661-71.

25. Urry J. The complexity turn. Theory, Culture and Society. 2005;22(5):1-14

26. Smith J, Jenks C. Qualitative Complexity: Ecology, Cognitive Processes and Re-emergence of Structures in Post-Humanist Social Theory. New York (NY): Routledge; 2006.

27. Cilliers P. Complexity and Postmodernism: Understanding Complex Systems. London (UK): Routledge; 1998.

28. Walby S. Complexity theory, systems theory, and multiple intersecting social inequalities. Philosophy of the Social Sciences. 2007;3(49).
29. Nowotny $\mathrm{H}$. The increase of complexity and its reduction: emergent interfaces between the natural sciences, humanities and social sciences. Theory, Culture and Society. 2005;22(5):15-31.

30. Shiell A, Hawe P, Gold L. Complex interventions or complex systems? Implications for health economic evaluation. BMJ. 2008;336:1281-3.

31. Byrne D. Social Exclusion. Berkshire (UK): Open University Press; 2005.

32. Blackman T. Placing Health: Neighbourhood Renewal, Health Improvement and Complexity. Bristol (UK): The Policy Press; 2006.

33. Hawe P, Sheill A, Riley T. Theorising Interventions as Events in Systems. Am J Community Psychol. 2009;43:267-76.

34. Howden-Chapman P, Crane J, Matheson A, Viggers H, Cunningham M, Blakely $\mathrm{T}$, et al. Retrofitting houses with insulation to reduce health inequalities: Aims and methods of a clustered, randomised community-based trial. Soc Sci Med. 2005;61:2600-10.

35. Blackman T. Complexity theory and the new public management. Social Issues. $2001 ; 1(2)$.

36. Byrne D. Complexity Theory and the Social Sciences. London (UK): Routlege; 1998.

37. Rickles D, Hawe P, Shiell A. A simple guide to chaos and complexity. J Epidemiol Community Health. 2007;61:933-7.

38. Say L, Raine R. A systematic review of inequalities in the use of maternal health care in developing countries: examining the scale of the problem and the importance of context. Bull World Health Organ. 2007;85(10).

\section{Authors}

Anna Matheson, Centre for Public Health Research, Massey University, Wellington, New Zealand Kevin Dew, School of Social and Cultural Studies, Victoria University of Wellington, New Zealand Jackie Cumming, Health Services Research Centre, Victoria University of Wellington, New Zealand

\section{Correspondence}

Dr Anna Matheson, Centre for Public Health Research, P.O. Box 756, Massey University, Wellington, New Zealand. Fax: +64 4380 0600; e-mail: a.g.matheson@massey.ac.nz 